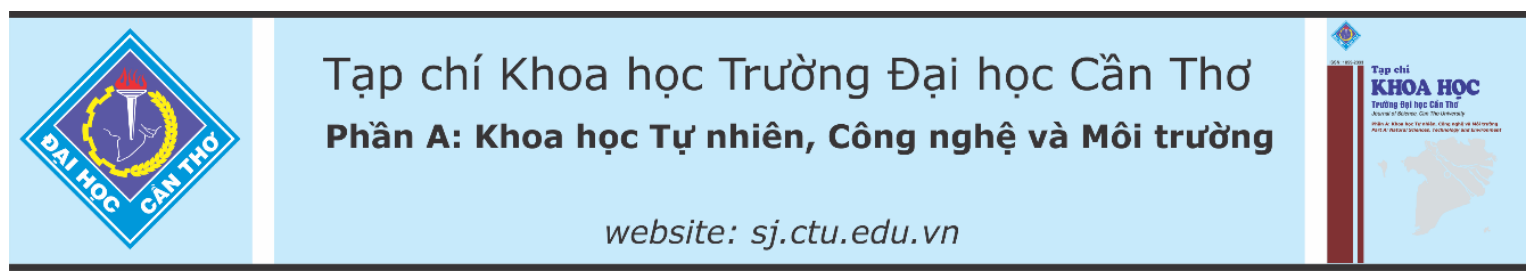

DOI:10.22144/ctu.jvn.2021.143

\title{
TÍNH CHẤT ĐIỀ TỬ CỦA DÃY NANO PENTA-GRAPHENE BIÊN RĂNG CƯA SAI HỎNG DẠNG KHUYẾT
}

\author{
Nguyễn Thành Tiên ${ }^{1 *}$, Lê Võ Phương Thuận ${ }^{1}$, Đào Hoàng Chương ${ }^{1}$ và Võ Thị Ngọc Huyền ${ }^{2}$ \\ ${ }^{1}$ Khoa Khoa học Tự nhiên, Truờng Đại học Cần Tho \\ ${ }^{2}$ Khoa Su phạm, Truờng Đại hoc Cần Tho \\ *Người chịu trách nhiệm về bài viết: Nguyễn Thành Tiên (email: nttien@ctu.edu.vn)
}

\section{Thông tin chung:}

Ngày nhận bài: 15/04/2021

Ngày nhận bài sủa: 05/06/2021

Ngày duyệt đăng: 29/10/2021

Title:

The electronic properties of defective sawtooth penta-graphene nanoribbons

\section{Tù khóa:}

Dãy nano penta-graphene, nguyên lý ban đầu, sai hỏng, tính chất điện tử

\section{Keywords:}

Defect, electronic properties, first principles, pentagraphene nanoribbon

\begin{abstract}
In this paper, the electronic properties of defective Sawtooth PentaGraphene Nanoribbons (DSSPGNRs) were investigated by calculating the binding energy, band structure, and density of states from first principles method. The types of defects considered here are monovacancy at either 4-coordinated C1 site or 3-coordinated C2 site and $2 \mathrm{C}$ double vacancy. The results showed that DSSPGNR has a significantly reduced band gap compared to the original sample. In which, the band gap of double DSSPGNR is more reducer than the one of single DSSPGNR. These findings are expected to provide important guidelines for the practical applications of penta-graphene.
\end{abstract}

\section{TÓM TẮT}

Trong bài báo này, tính chất điện tử của các dãy penta-graphene dạng răng cưa (SSPGNR) sai hỏng dạng khuyết (DSSPGNRs) được nghiên cứu bằng cách tính năng luợng liên kết, cấu trúc vùng điện tử và mật độ trạng thái bởi phuơng pháp nguyên lý ban đầu. Ba kiểu khuyết được khảo sát trong nghiên cúu này là khuyết đơn nguyên tử $C 1, C 2$ và khuyết đồng thời hai nguyên tủ $\mathrm{C} 2$. Kết quả nghiên cứu cho thấy DSSPGNR có độ rộng vùng cấm giảm đáng kể so với mẫu không khuyết. Trong đó, DSSPGNRs khuyết đồng thời hai nguyên tủ $\mathrm{C} 2$ có độ rộng vùng cấm giảm nhiều hơn so với DSSPGNRs khuyết đơn nguyên tư. Kết quả nghiên cúu này cung cấp thông tin quan trọng cho việc phát triển úng dụng penta-graphene trong lĩnh vục vi điện tử.

\section{GIÓ́I THIỆU}

Trong số các vật liệu hai chiều, Graphene và các vật liệu tựa Graphene đã nhanh chóng thu hút sự chú ý của cộng đồng các nhà khoa học vật liệu do tính chất vật lý thay đổi đáng kể của chúng so với cấu trúc khối. Chúng có nhiều tiềm năng cho các ứng dụng trong các thiết bị điện tử thế hệ mới bởi vì tính chất vận chuyển điện tử độc đáo như: độ linh động hạt tải cao, dẫn nhiệt tốt và các hiệu ứng Hall lượng tử dị thường (Berger et al., 2004; Bolotin et al.,
2008; Hirata et al., 2004; Neto et al., 2009). Sự lai hóa $\mathrm{sp}^{2}$ của liên kết Carbon và vùng cấm điện tử bằng 0 của graphene làm nó không hiệu quả đối với hấp phụ khí và ứng dụng phát triển transistor, sensors.

Gần đây, Penta-Graphene (PG), một cấu trúc các nguyên tử $\mathrm{C}$ đơn lớp xếp hình ngũ giác đã được đề xuất. Cấu trúc $\mathrm{PG}$ là tổ hợp của các nguyên tử $\mathrm{C} 1$ (lai hóa $\mathrm{sp}^{3}$ ) và các nguyên tử $\mathrm{C} 2$ (lai hóa $\mathrm{sp}_{2}$ ) (Zhang et al., 2015). Các nghiên cứu tiên đoán 
Graphene là một bán dẫn có vùng cấm gián tiếp với độ rộng vùng cấm là $3.25 \mathrm{eV}$ (Wang et al., 2016; Yu \& Zhang 2015; Zhang et al., 2015 ). Bên cạnh đó, Graphene có hệ số Poisson âm bất thường, độ bền siêu cao và khả năng chịu được nhiệt cao tới 1000 $K$, vượt trội Graphene. Những đặc tính vượt trội này làm cho nó trở thành một ứng cử viên tiềm năng cho nhiều ứng dụng đầy hứa hẹn. Nhiều nghiên cứu được thực hiện để cải thiện các tính chất vật lý của PG thông qua các phương pháp chức năng hóa khác nhau. Các nghiên cứu lý thuyết cho thấy PG có độ dẫn nhiệt gia tăng đáng kể sau khi hydro hóa, cao hơn nhiều so với $\mathrm{PG}$ thuần. Nguyên tử Hydro hấp phụ trên tấm $\mathrm{PG}$ được dự đoán sẽ tạo ra từ tính và có thể điều chỉnh hiệu quả các tính chất điện tử của PG như việc điều chỉnh $\mathrm{PG}$ từ một chất bán dẫn thành một chất bán kim. Ngoài ra, trong cấu trúc của $\mathrm{PG}$ có chứa cả liên kết Carbon $\mathrm{sp}^{2}$ và $\mathrm{sp}^{3}$ vì vậy $\mathrm{PG}$ không có cấu trúc phẳng lý tưởng, mà nó có cấu trúc vênh, cho thấy PG có nhiều vị trí sở hữu khả năng hấp phụ khí (Zhang et al., 2015). Những đặc tính này khiến cho $\mathrm{PG}$ trở thành ứng cử viên tiềm năng để ứng dụng trong lĩnh vực vi điện tử.

Nhằm mục đích giảm kích thước linh kiện, tấm PG được cắt thành các cấu trúc giả một chiều được gọi là Penta-Graphene nanoribbon (PGNR). PGNR có 4 loại: $\mathrm{ZZ}, \mathrm{AA}, \mathrm{ZA}, \mathrm{SS}$. Trong đó, SS là loại đáng chú ý nhất không chỉ bởi vì nó là bán dẫn mà còn vì nó là cấu trúc bền nhất trong bốn loại PGNR. Sự hấp phụ các phân tử khí $\mathrm{CO}, \mathrm{CO}_{2}, \mathrm{NH}_{3}$ trên bề mặt cấu trúc này được nghiên cứu, xác định được đặc tính hấp phụ của chúng (Nguyễn Thành Tiên và ctv., 2020). Nghiên cứu tính chất vận chuyển điện tử của
SSPGNR pha tạp thay thế (Si, P, N) đã được thực hiện, kết quả cho thấy cấu trúc vùng năng lượng và đặc trưng $\mathrm{I}-\mathrm{V}$ của $\mathrm{N}$ :SSPGNR thay đổi đáng kể so với mẫu nguyên sơ (Tien et al., 2019). Tuy nhiên, ta biết rằng, các cấu trúc thấp chiều nói chung dễ bị sai hỏng khuyết khi tổng hợp chúng (Banhart et al., 2011; Han et al., 2021). Vì thế, việc nghiên cứu đặc tính điện tử các cấu trúc khuyết là rất cần thiết, có thêm thông tin về cấu trúc thực. Trong nghiên cứu này, dựa trên cơ sở lý thuyết phiếm hàm mật độ, sự tồn tại và đặc tính điện tử của các SSPGNR sai hỏng khuyết được nghiên cứu.

\section{PHƯƠNG PHÁP}

Sự ổn định cấu trúc và đặc tính điện tử của các mẫu SSPGNR khuyết được đánh giá bằng phương pháp nguyên lý ban đầu dựa trên lý thuyết phiếm hàm mật độ DFT (Kohn \& Sham, 1965). Mẫu nghiên cứu có độ rộng thay đổi từ năm đến tám chuỗi được cắt theo biên răng cưa. Các mẫu SSPGNR với các vị trí khuyết khác nhau được tối ưu bằng cách sử dụng tính toán DFT trong phép tính gần đúng gradient tổng quát (GGA) của PerdewBurke-Ernzerhof (PBE) (Perdew et al., 1996) trong gói phần mềm Castep để tìm được các cấu trúc ổn định. Số k-point trong vùng Brillouin được chọn là $1 \mathrm{x} 1 \mathrm{x} 5$ lần lượt theo ba phương $\mathrm{x}, \mathrm{x}, \mathrm{z}$ với năng lượng cutoff là $680 \mathrm{eV}$ và nhiệt độ động học của hệ điện tử là $300 \mathrm{~K}$. Trong quá trình tối ưu, tiêu chí hội tụ về lực và ứng suất tối đa có giá trị lần lượt là $0,05 \mathrm{eV} / \AA ̊$ và $0,1 \mathrm{Gpa}$.

\section{KẾT QUẢ VÀ THẢO LUẬN}

\subsection{Sự ổn định cấu trúc}
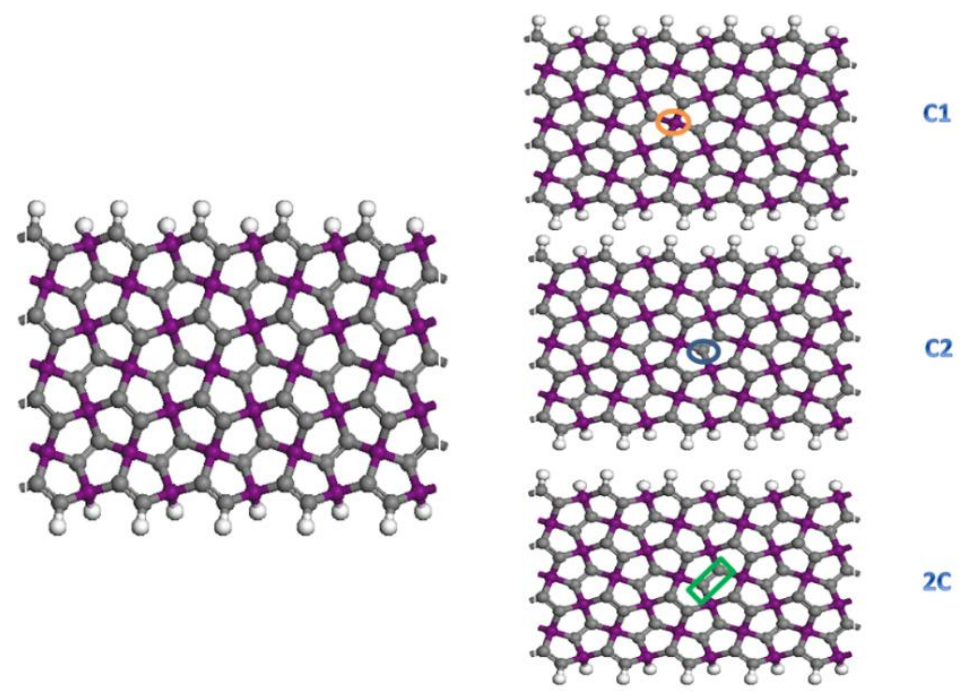

Hình 1. Hình bên trái : Mô hình SSPGNR không khuyết; Hình bên phải: Mô hình SSPGNR với vị trí của nguyên tử có khả năng bị khuyết: $\mathrm{C} 1, \mathrm{C} 2$ và hai nguyên tử $\mathrm{C} 2(2 \mathrm{C})$ 
Đầu tiên, cấu hình ổn định nhất được tìm trong các trường hợp khuyết khả dĩ trên SSPGNR giả một chiều. Để tìm được các vị trí khuyết khả dĩ, các mẫu SSPGNR được khảo sát với độ rộng dãy thay đổi từ 5 đến 8 dãy, trên mỗi mẫu đã tìm thấy có 3 cấu trúc khuyết khả dĩ (được trình bày trong Hình 1). Hai cấu trúc khả dĩ đầu tiên của SSPGNR là khuyết một nguyên tử Carbon $\mathrm{C} 1$ và $\mathrm{C} 2$. Cấu trúc khả dĩ thứ ba là khuyết hai nguyên tử $\mathrm{C} 2$. Các cấu trúc khuyết khả dĩ sau tối ưu được mô tả trong Hình 2 (độ rộng 5, 6 dãy) và Hình 3 (độ rộng 7,8 dãy). a)

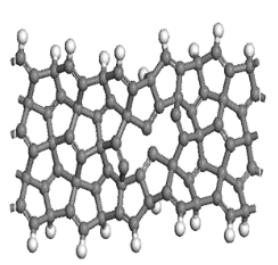

d)
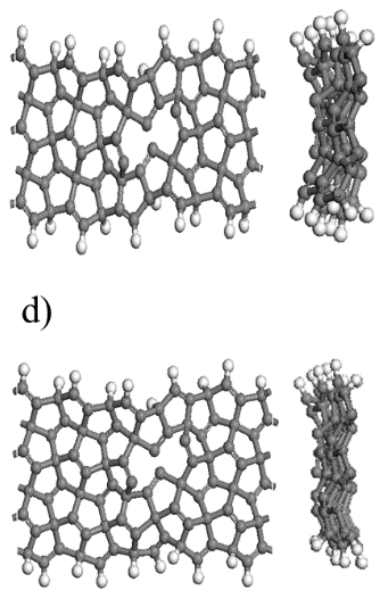

b)

e)

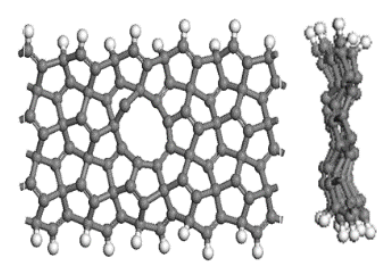

c)
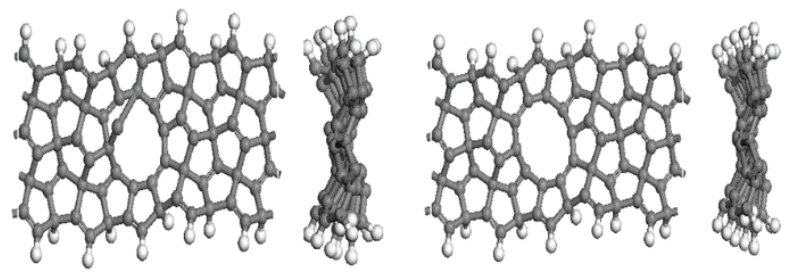

f)

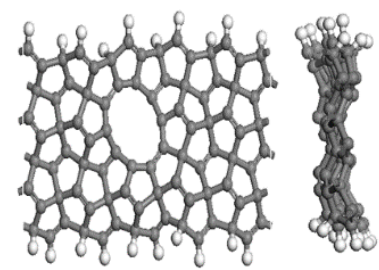

Hình 2. Các cấu hình bị khuyết khả dĩ của các SSPGNR có độ rộng 5 và 6 dãy: (a), (d) cấu hình khuyết nguyên tử C1 (SS5_C1 và SS6_C1), (b), (e) cấu hình khuyết nguyên tử C2 (SS5_C2 và SS6_C2) và $(c)$, (f) cấu hình khuyết cả hai nguyên tử C2 (SS5_2C và SS6_2C) trên SSPGNR

a)

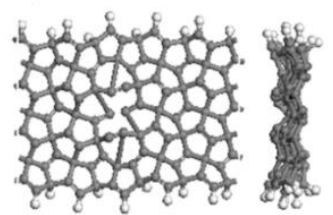

d)

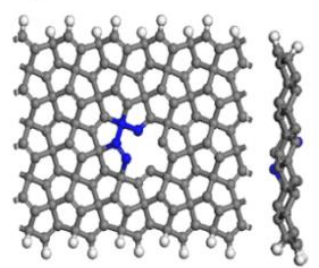

b)

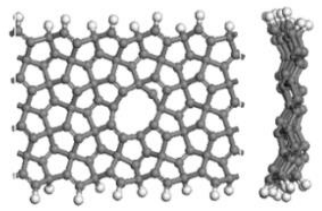

e)

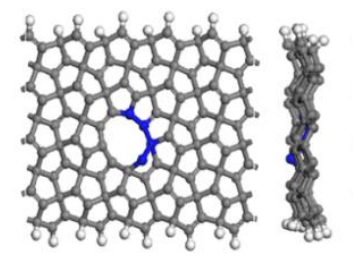

c)

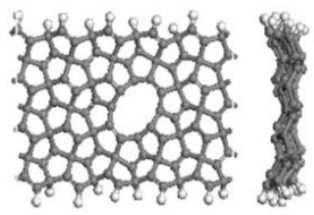

f)

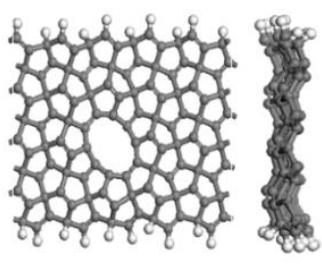

Hình 3. Các cấu hình bị khuyết khả dĩ của các SSPGNR có độ rộng 7 và 8 dãy: (a), (d) cấu hình khuyết nguyên tử C1 (SS7 C1 và SS8 C1), (b), (e) cấu hình khuyết nguyên tử C2 (SS7C2 và SS8 C2) và $(c)$, (f) cấu hình khuyết cả hai nguyên tử C2 (SS7_2C và SS8_2C) trên SSPGNR

Các mẫu được tối ưu trên cơ sở ổn định năng lượng tổng của hệ (năng lượng hệ thấp nhất). Để đánh giá cẩu trúc ổn định nhất trong tất cả các trường hợp nghiên cứu, năng lượng liên kết $E_{\text {bind }}$ của
SSPGNR bị khuyết được tính toán. Năng lượng $E_{\text {bind }}$ được tính dựa trên công thức sau:

$$
E_{\text {bind }}=\frac{E_{\text {total }}-N_{C} E_{C}-N_{H} E_{H}}{N_{C}+N_{H}}
$$


trong đó $E_{\text {total }}, E_{C}$ và $E_{H}$ lần lượt là tổng năng lượng của hệ, năng lượng của nguyên tử Carbon, năng lượng của nguyên tử Hydro cô lập. $\mathrm{N}_{\mathrm{C}}$ và $\mathrm{N}_{\mathrm{H}}$ lần lượt là số nguyên tử Carbon và Hydro trong hệ. Năng lượng liên kết càng nhỏ thì cấu hình khuyết tương ứng được xem là càng ổn định. Kết quả tính năng lượng liên kết được trình bày trong Bảng 1 . Kết quả tính toán cho thấy các mẫu khuyết SS8 có năng lượng liên kết thấp nhất. Giá trị năng lượng liên kết của mẫu khuyết SS8 giảm dần theo thứ tự: $\mathrm{E}_{\text {bind }}\left(\mathrm{SS} 8 \_\mathrm{C} 1\right)=-7,374 \mathrm{eV}>\mathrm{E}_{\text {bind }}\left(\mathrm{SS} 7 \_2 \mathrm{C}\right)=$ 7,381 eV> E bind $_{\text {(SS7_C2) }}=-7,421 \mathrm{eV}$. Tuy nhiên, nếu xét cùng loại nguyên tử khuyết thì mẫu SS7_C1 ổn định hơn các mẫu khuyết $\mathrm{Cl}$ còn lại vì mẫu này đối xứng hơn và rộng hơn.

Bảng 1. Năng lượng liên kết (Ebind) của các DSSPGNRs

\begin{tabular}{|c|c|c|c|c|c|c|}
\hline \multirow{2}{*}{ Mẫu } & \multirow{2}{*}{ Vị trí } & \multicolumn{5}{|c|}{$E_{\text {bind }}(\mathrm{eV})$} \\
\hline & & SS5 & SS6 & SS7 & $S S 8$ & $P G$ \\
\hline$\overline{C 1}$ & $C 1\left(s p^{3}\right)$ & $-7,102$ & $-7,189$ & $-7,383$ & $-7,374$ & $-6,964$ \\
\hline$C 2$ & $C 2\left(s p^{2}\right)$ & $-7,123$ & $-7,207$ & $-7,295$ & $-7,421$ & $-7,033$ \\
\hline $2 C$ & $C 2+C 2$ & $-7,157$ & $-7,246$ & $-7,363$ & $-7,381$ & \\
\hline
\end{tabular}

3.2. Các thông số hình học của cấu trúc

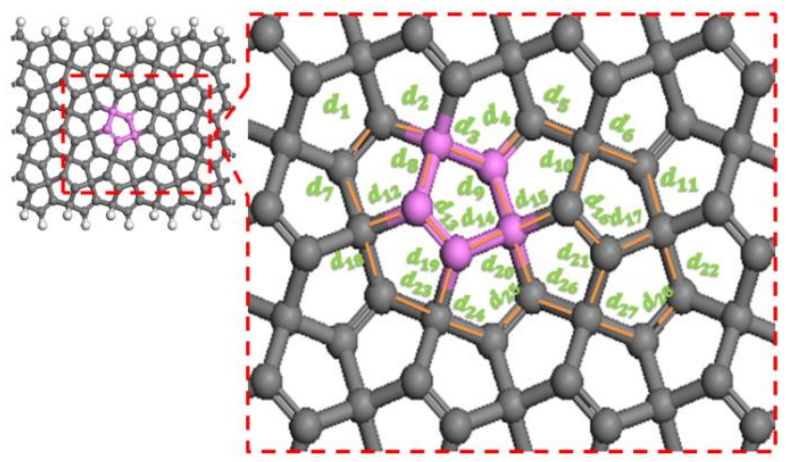

Hình 4. Mô hình minh họa độ dài các liên kết của SSPGNR quanh vị trí khuyết

Sau khi tối ưu, các thông số hình học được xác định. Hình 4 ký hiệu các vị trí liên kết xung quanh nguyên tử khuyết. Bảng 2 , Bảng 3 , Bảng 4 và Bảng 5 trình bày các thông số hình học là các độ dài liên kết của các DSSPGNR sau khi tối ưu cấu trúc. Có thể thấy rằng, độ dài liên kết giữa các nguyên tử xung quanh nguyên tử khuyết có sự thay đổi đáng kể. Trong đó, các mẫu bị khuyết tại vị trí $\mathrm{C} 1$ có liên kết bị kéo dài nhiều nhất và mẫu $2 \mathrm{C}$ có liên kết ít thay đổi nhất. Đây cũng là nguyên nhân chính khiến năng lượng liên kết trong các mẫu $\mathrm{C} 1$ là lớn nhất, trừ mẫu SS7_C1.

Bảng 2. Các độ dài liên kết của SS5 không khuyết và khuyết

\begin{tabular}{rcccc|ccccc}
\hline & SS5 & SS5_C1 & SS5_C2 & SS5_2C & & SS5 & SS5_C1 & SS5_C2 & SS5_2C \\
\hline d1 & 1,337 & 1,343 & 1,448 & 1,436 & $\mathbf{d 1 5}$ & 1,541 & $\ldots$ & 1,431 & 1,433 \\
d2 & 1,539 & 1,561 & 1,392 & 1,358 & $\mathbf{d 1 6}$ & 1,337 & 1,337 & 1,358 & 1,358 \\
$\mathbf{d 3}$ & 1,541 & 1,619 & 1,425 & 1,398 & $\mathbf{d 1 7}$ & 1,539 & 1,579 & 1,564 & 1,565 \\
$\mathbf{d 4}$ & 1,337 & 1,455 & 1,378 & 1,529 & $\mathbf{d 1 8}$ & 1,554 & 1,521 & 1,492 & 1,381 \\
$\mathbf{d 5}$ & 1,539 & 1,529 & 1,536 & 1,526 & $\mathbf{d 1 9}$ & 1,547 & 1,502 & $\ldots$ & $\ldots$ \\
d6 & 1,541 & 1,446 & 1,557 & 1,340 & $\mathbf{d 2 0}$ & 1,553 & $\ldots$ & 1,381 & 1,464 \\
d7 & 1,553 & 1,514 & 1,480 & 1,464 & $\mathbf{d 2 1}$ & 1,547 & 1,587 & 1,632 & 1,548 \\
d8 & 1,552 & 1,588 & 2,598 & $\ldots$ & $\mathbf{d 2 2}$ & 1,553 & 1,514 & 1,537 & 1,564 \\
d9 & 1,552 & $\ldots$ & 1,446 & 1,381 & $\mathbf{d 2 3}$ & 1,539 & 1,440 & 1,435 & 1,398 \\
$\mathbf{d 1 0}$ & 1,552 & 1,502 & 1,552 & 1,631 & $\mathbf{d 2 4}$ & 1,548 & 1,529 & 1,357 & 1,358 \\
$\mathbf{d 1 1}$ & 1,553 & 1,521 & 1,567 & 1,535 & $\mathbf{d 2 5}$ & 1,338 & 1,455 & 1,398 & 1,436 \\
$\mathbf{d 1 2}$ & 1,541 & 1,580 & 1,507 & $\ldots$ & $\mathbf{d 2 6}$ & 1,539 & 1,619 & 1,530 & 1,372 \\
$\mathbf{d 1 3}$ & 1,337 & 1,337 & $\ldots$ & $\ldots$ & $\mathbf{d 2 7}$ & 1,548 & 1,561 & 1,526 & 1,537 \\
$\mathbf{d 1 4}$ & 1,539 & $\ldots$ & $\ldots$ & $\ldots$ & $\mathbf{d 2 8}$ & 1,338 & 1,343 & 1,340 & 1,555 \\
\hline
\end{tabular}


Bảng 3. Các độ dài liên kết của SS6 không khuyết và khuyết

\begin{tabular}{lllll|lllll}
\hline & SS6 & SS6_C1 & SS6_C2 & SS6_2C & & SS6 & SS6_C1 & SS6_C2 & SS6_2C \\
\hline d1 & 1,337 & 1,340 & 1,368 & 1,387 & d15 & 1,541 & $\ldots$ & 1,431 & 1,438 \\
d2 & 1,539 & 1,562 & 1,465 & 1,359 & d16 & 1,337 & 1,340 & 1,352 & 1,354 \\
d3 & 1,541 & 1,654 & 1,497 & 1,448 & $\mathbf{d 1 7}$ & 1,539 & 1,573 & 1,543 & 1,542 \\
d4 & 1,337 & 1,453 & 1,363 & 1,376 & d18 & 1,554 & 1,509 & 1,512 & 1,454 \\
d5 & 1,539 & 1,535 & 1,548 & 1,540 & $\mathbf{d 1 9}$ & 1,547 & 1,446 & $\ldots$ & $\ldots$ \\
d6 & 1,541 & 1,446 & 1,571 & 1,564 & $\mathbf{d 2 0}$ & 1,553 & $\ldots$ & 1,379 & 1,394 \\
d7 & 1,553 & 1,509 & 1,525 & 1,373 & $\mathbf{d 2 1}$ & 1,547 & 1,581 & 1,603 & 1,601 \\
d8 & 1,552 & 1,547 & 1,617 & $\ldots$ & $\mathbf{d 2 2}$ & 1,553 & 1,507 & 1,533 & 1,531 \\
d9 & 1,552 & $\ldots$ & 1,470 & 1,482 & $\mathbf{d 2 3}$ & 1,539 & 1,441 & 1,459 & 1,444 \\
d10 & 1,552 & 1,502 & 1,545 & 1,549 & $\mathbf{d 2 4}$ & 1,548 & 1,499 & 1,370 & 1,367 \\
d11 & 1,553 & 1,526 & 1,571 & 1,567 & $\mathbf{d 2 5}$ & 1,338 & 1,477 & 1,395 & 1,410 \\
d12 & 1,541 & 1,606 & 1,579 & $\ldots$ & $\mathbf{d 2 6}$ & 1,539 & 1,640 & 1,522 & 1,542 \\
d13 & 1,337 & 1,343 & $\ldots$ & $\ldots$ & d27 & 1,548 & 1,662 & 1,575 & 1,593 \\
d14 & 1,539 & $\ldots$ & $\ldots$ & $\ldots$ & d28 & 1,338 & 1,344 & 1,341 & 1,343 \\
\hline
\end{tabular}

Bảng 4. Các độ dài liên kết của SS7 không khuyết và khuyết

\begin{tabular}{lllll|lllll}
\hline & SS7 & SS7_C1 & SS7_C2 & SS7_2C & & SS7 & SS7_C1 & SS7_C2 & SS7_2C \\
\hline d1 & 1,539 & 1,469 & 1,552 & 1,545 & d15 & 1,540 & $\ldots$ & $\ldots$ & $\ldots$ \\
d2 & 1,548 & 1,398 & 1,571 & 1,558 & d16 & 1,337 & 1,356 & $\ldots$ & $\ldots$ \\
d3 & 1,337 & 1,234 & 1,364 & 1,375 & d17 & 1,539 & 1,727 & 1,551 & $\ldots$ \\
d4 & 1,539 & 1,482 & 1,504 & 1,454 & $\mathbf{d 1 8}$ & 1,551 & 1,564 & 1,534 & 1,531 \\
d5 & 1,547 & 1,524 & 1,526 & 1,371 & $\mathbf{d 1 9}$ & 1,546 & 1,599 & 1,597 & 1,600 \\
d6 & 1,337 & 1,332 & 1,372 & 1,398 & $\mathbf{d 2 0}$ & 1,551 & $\ldots$ & 1,382 & 1,387 \\
d7 & 1,551 & 1,552 & 1,564 & 1,559 & $\mathbf{d 2 1}$ & 1,546 & 2,890 & $\ldots$ & $\ldots$ \\
d8 & 1,546 & 2,890 & 1,507 & 1,515 & $\mathbf{d 2 2}$ & 1,551 & 1,552 & 1,522 & 1,469 \\
d9 & 1,551 & $\ldots$ & 1,469 & 1,469 & $\mathbf{d 2 3}$ & 1,337 & 1,332 & 1,339 & 1,341 \\
d10 & 1,546 & 1,599 & 1,548 & $\ldots$ & $\mathbf{d 2 4}$ & 1,547 & 1,525 & 1,573 & 1,587 \\
d11 & 1,551 & 1,563 & 1,539 & 1,386 & $\mathbf{d 2 5}$ & 1,539 & 1,482 & 1,521 & 1,530 \\
d12 & 1,540 & 1,729 & 1,552 & 1,549 & $\mathbf{d 2 6}$ & 1,337 & 1,234 & 1,391 & 1,398 \\
d13 & 1,337 & 1,356 & 1,354 & 1,351 & $\mathbf{d 2 7}$ & 1,547 & 1,397 & 1,373 & 1,371 \\
d14 & 1,539 & $\ldots$ & 1,424 & 1,427 & d28 & 1,539 & 1,469 & 1,463 & 1,454 \\
\hline
\end{tabular}

Bảng 5. Các độ dài liên kết của SS8 không khuyết và khuyết

\begin{tabular}{|c|c|c|c|c|c|c|c|c|c|}
\hline & SS8 & SS8_C1 & SS8_C2 & SS8_2C & & SS8 & SS8_C1 & SS8_C2 & SS8_2C \\
\hline d1 & 1,337 & 1,335 & 1,389 & 1,400 & d15 & 1,541 & & 1,544 & 1,428 \\
\hline d2 & 1,539 & 1,532 & 1,369 & 1,369 & d16 & 1,337 & 1,324 & 1,341 & 1,351 \\
\hline d3 & 1,541 & 1,550 & 1,459 & 1,456 & d17 & 1,539 & 1,595 & 1,537 & 1,552 \\
\hline d4 & 1,337 & 1,328 & 1,368 & 1,377 & d18 & 1,554 & 1,524 & 1,465 & 1,473 \\
\hline d5 & 1,539 & 1,527 & 1,544 & 1,546 & d19 & 1,547 & 1,547 & 1,550 & . . \\
\hline d6 & 1,541 & 1,535 & ... & 1,543 & d20 & 1,553 & ... & 1,535 & 1,389 \\
\hline d7 & 1,553 & 1,538 & 1,380 & 1,389 & d21 & 1,547 & 1,535 & 1,554 & 1,599 \\
\hline d8 & 1,552 & 1,540 & $\ldots$ & .. & d22 & 1,553 & 1,539 & 1,549 & 1,536 \\
\hline d9 & 1,552 & $\ldots$ & 1,519 & 1,472 & d23 & 1,539 & 1,533 & 1,501 & 1,456 \\
\hline d10 & 1,552 & 1,546 & 1,551 & 1,518 & d24 & 1,548 & 1,531 & 1,525 & 1,372 \\
\hline d11 & 1,553 & 1,525 & 1,550 & 1,564 & d25 & 1,338 & 1,323 & 1,372 & 1,400 \\
\hline d12 & 1,541 & 1,596 & $\ldots$ & $\ldots$ & d26 & 1,539 & 1,549 & 1,545 & 1,531 \\
\hline d13 & 1,337 & 1,324 & $\ldots$ & $\ldots$ & d27 & 1,548 & 1,542 & 1,552 & 1,583 \\
\hline d14 & 1,539 & & 1,551 & $\ldots$ & d28 & 1,338 & 1,337 & 1,338 & 1,342 \\
\hline
\end{tabular}




\subsection{Cấu trúc vùng năng lượng (BS)}

Để đánh giá sự ảnh hưởng của các vị trí khuyết khác nhau lên đặc tính điện tử của SSPGNR, sự thay

a)
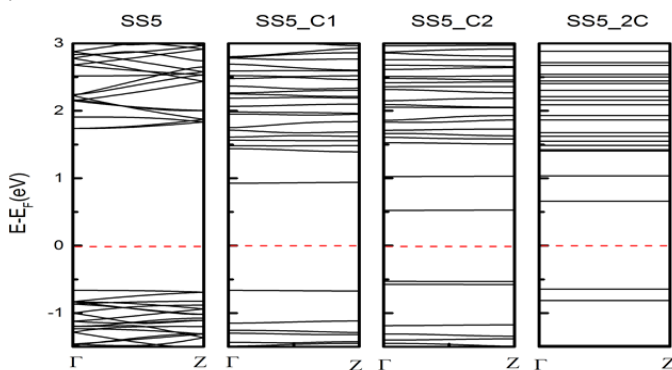

c)
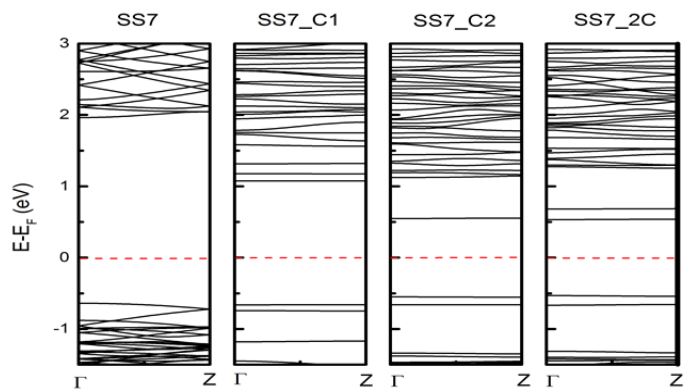

đổi của cấu trúc vùng năng lượng của SSPGNR và DSSPGNR cần được xem xét. Hình 5 mô tả cấu trúc vùng năng lượng của các mẫu: (a) SS5, (b) SS6, (c) SS7 và (d) SS8 trước và sau khi khuyết.

b)

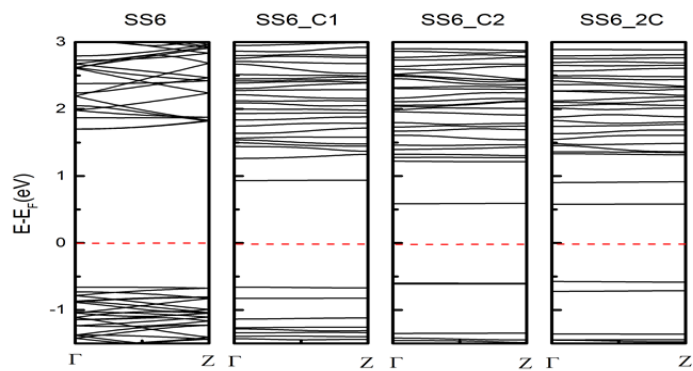

d)

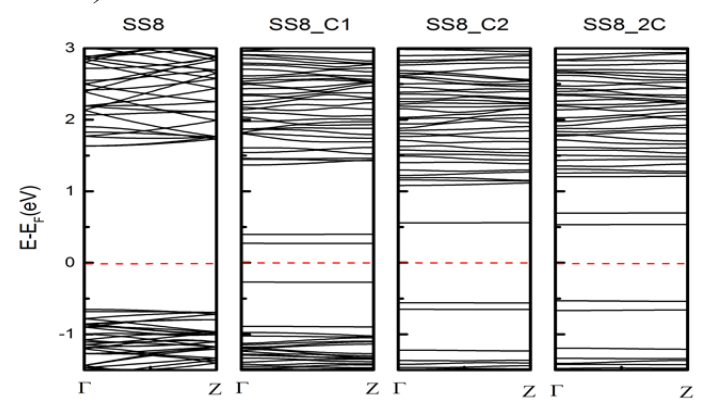

Hình 5. Cấu trúc vùng năng lượng của các cấu trúc SSPGNR không khuyết và khuyết: (a) SS5, (b) SS6, (c) SS7 và (d) SS8. Đường không liền nét biểu diễn mức năng lượng Fermi

Kết quả cho thấy sau khi bị khuyết tất cả các mẫu đều có độ rộng vùng cấm bị giảm đi đáng kể. Trước khi bị khuyết, SSPGNR có độ rộng vùng cấm vào khoảng $2,2 \mathrm{eV}$ đến $2,4 \mathrm{eV}$ tùy vào độ rộng dãy của cấu trúc nhưng sau khi bị khuyết độ rộng vùng cấm đa số mẫu giảm xuống cỡ khoảng $1 \mathrm{eV}$. Trong đó, mẫu SS6 và SS7 bị khuyết tại vị trí $2 \mathrm{C}$ có độ rộng vùng cấm giảm nhiều nhất. Mẫu SS5 bị khuyết tại vị trí $\mathrm{C} 2$ có vùng cấm giảm nhiều nhất. Tuy nhiên, với mẫu độ rộng dãy $\mathrm{W}=8$ khuyết tại vị trí $\mathrm{C} 1$ có độ rộng vùng cấm giảm nhiều hơn $(0,541 \mathrm{eV})$. Điều này tương tự với kết quả của nghiên cứu trước về mẫu PG hai chiều khuyết (DPG) (Lima et al., 2021).

Theo kết quả tính từ Bảng 6 , các mẫu khuyết vẫn thuộc vật liệu bán dẫn nhưng có độ rộng vùng cấm giảm đi. Một đặc điểm đáng chú ý, các vùng con của các mẫu khuyết quanh mức Fermi có dạng thẳng, điều này thể hiện hiệu ứng giam cầm điện tử định xứ quanh vị trí khuyết, điện tử có năng lượng này thể hiện đặc tính giả không chiều. Vậy đặc tính động học điện tử trong khoảng năng lượng lân cận năng lượng Fermi của các mẫu khuyết có tính chất giả không chiều. Điều này hứa hẹn một số tính chất vật lý mới của mẫu khuyết so với mẫu không khuyết.

Bảng 6. Độ rộng vùng cấm $(\mathrm{Eg})$ của DSSPGNR

\begin{tabular}{ccc}
\hline Mẫu & Vị trí khuyết & $\mathbf{E}_{\mathbf{g}}(\mathbf{e V})$ \\
\hline \multirow{3}{*}{ PG } & Không khuyết & 2,369 \\
& $\mathrm{C} 1$ & 0,491 \\
& $\mathrm{C} 2$ & 1,094 \\
\hline \multirow{3}{*}{ SS5 } & Không khuyết & 2,401 \\
& $\mathrm{C} 1$ & 1,590 \\
& $\mathrm{C} 2$ & 1,053 \\
& 2C & 1,303 \\
\hline \multirow{3}{*}{ SS6 } & Không khuyết & 2,359 \\
& $\mathrm{C} 1$ & 1,592 \\
& $\mathrm{C} 2$ & 1,185 \\
& $2 \mathrm{C}$ & 1,154 \\
\hline \multirow{3}{*}{ SS7 } & Không khuyết & 2,325 \\
& $\mathrm{C} 1$ & 1,414 \\
& $\mathrm{C} 2$ & 1,098 \\
& 2C & 1,065 \\
\hline \multirow{3}{*}{ SS8 } & Không khuyết & 2,286 \\
& $\mathrm{C} 1$ & 0,541 \\
& $\mathrm{C} 2$ & 1,116 \\
& $2 \mathrm{C}$ & 1,065 \\
\hline
\end{tabular}




\subsection{Mật độ trạng thái (DOS) của DSSPGNR}

Trong Hình 6, mật độ trạng thái của các mẫu DSSPGNR được tính với độ rộng dãy khác nhau: (a) SS5, (b) SS6, (c) SS7 và (d) SS8. Nhìn chung, các mẫu DSSPGNR vẫn là các chất bán dẫn nhưng độ rộng vùng cấm có thay đổi theo xu hướng giảm. Rõ ràng sự tồn tại của sai hỏng khuyết ảnh hưởng đến độ rộng vùng cấm là đáng kể. Kết quả tính DOS phù hợp với kết quả tính BS. Trong đó, mẫu SS5 bị khuyết tại vị trí $\mathrm{C} 2$ có vùng cấm giảm nhiều nhất. Các mẫu $\mathrm{SS} 6$ và $\mathrm{SS} 7$ có độ rộng vùng cấm giảm

a)

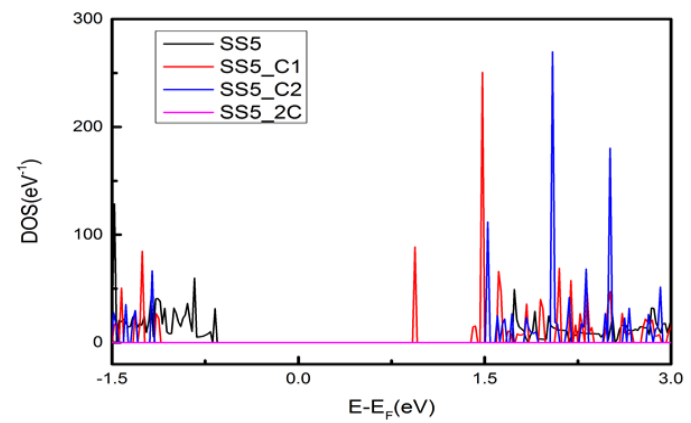

c)

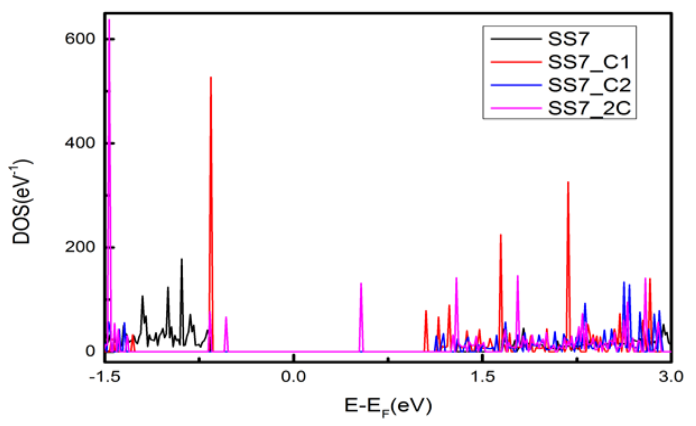

mạnh nhất khi mẫu bị khuyết cả hai nguyên tử Carbon $2 \mathrm{C}$ và giảm ít nhất khi mẫu bị khuyết nguyên tử $\mathrm{C} 1$. Đặc biệt, mẫu SS8 có độ rộng vùng cấm giảm nhiều nhất khi bị khuyết nguyên tử $\mathrm{C} 1$ và ít nhất khi bị khuyết nguyên tử $\mathrm{C} 2$. Đồ thị DOS thể hiện các mẫu DSSPGNR có mật độ trạng thái cao ở một số mức năng lượng quanh mức Fermi. Các mức năng lượng này có khả năng là cầu nối hữu hiệu khi chuyển dời quang điện tử với hiệu suất cao. Kết quả tính này cũng cho thấy mẫu DSSPGNR có đặc tính điện tử phong phú hơn DPG.

b)

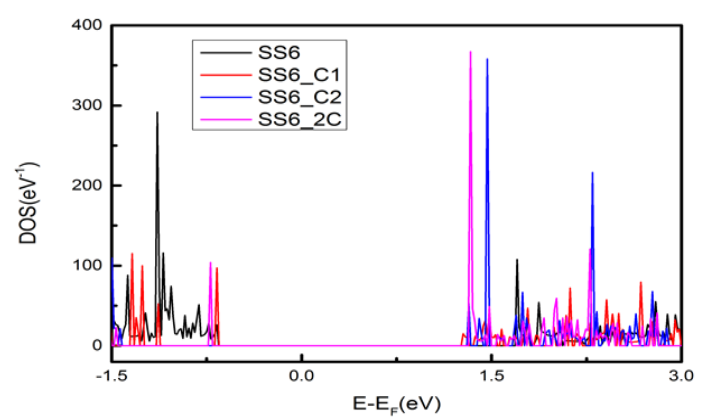

d)

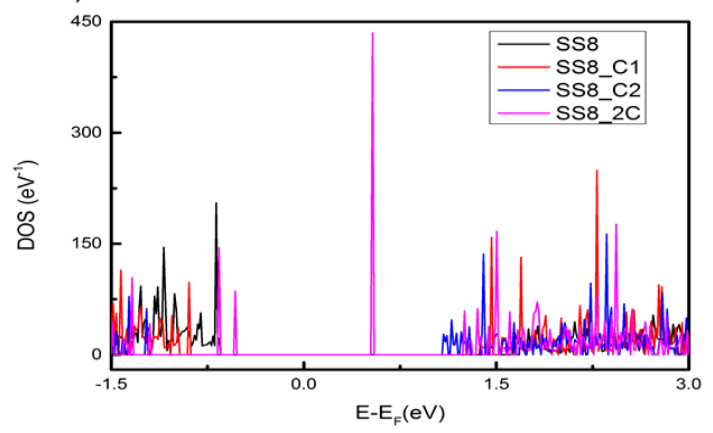

Hình 6. DOS của các mẫu DSSPGNR: a) rộng 5 chuỗi, b) rộng 6 chuỗi, c) rộng 7 chuỗi và d) rộng 8 chuỗi

\section{KẾT LUẬN}

Tóm lại, thông qua việc đánh giá sự ổn định cấu trúc và đặc tính điện tử của cấu trúc DSSPGNR với sự thay đổi độ rộng dãy bằng phương pháp nguyên lý ban đầu, dựa trên lý thuyết phiếm hàm mật độ, tính trên máy tính hiệu năng cao. Kết quả cho thấy rằng năng lượng liên kết của các mẫu SS8 nói chung có giá trị thấp nhất, chứng tỏ mẫu SS8 là ổn định nhất. Độ dài liên kết và góc liên kết ở tất cả các mẫu có sự thay đổi đáng kể xung quanh vị trí khuyết. Độ rộng vùng cấm của các mẫu DSSPGNR giảm đáng kể so với các mẫu SSPGNR cùng độ rộng dãy. Đặc biệt, xuất hiện các trạng thái điện tử mới dạng thẳng trong vùng cấm lân cận mức Fermi. Kết quả nghiên cứu này định hướng quan trọng cho việc xác định tính chất điện tử của cấu trúc SSPGNR không hoàn hảo; từ đó, định hướng cho việc phát triển các linh kiện quang điện tử dựa trên cấu trúc nghiên cứu.

\section{LờI CẢM TẠ}

Nghiên cứu này được tài trợ bởi đề tài khoa học và công nghệ cấp Bộ, mã số: B2020-TCT-14 và hướng dẫn sinh viên nghiên cứu khoa học trong khuôn khổ đề tài mã số TSV2021-.

\section{TÀI LIỆ THAM KHẢO}

Berger, C., Song, Z., Li, T., Li, X., Ogbazghi, A. Y., Feng, R., ... \& De Heer, W. A. (2004). Ultrathin epitaxial graphite: $2 \mathrm{D}$ electron gas properties and a route toward graphene-based 
nanoelectronics. The Journal of Physical Chemistry B, 108(52), 19912-19916.

Bolotin, K. I., Sikes, K. J., Jiang, Z., Klima, M., Fudenberg, G., Hone, J., ... \& Stormer, H. L. (2008). Ultrahigh electron mobility in suspended graphene. Solid state communications, 146(910), 351-355.

Banhart, F., Kotakoski, J., \& Krasheninnikov, A. V. (2011). Structural defects in graphene. ACS nano, 5(1), 26-41.

Han, T., Wang, X., Zhang, X., Scarpa, F., \& Tang, C. (2021). Mechanics of penta-graphene with vacancy defects under large amplitude tensile and shear loading. Nanotechnology, 32(27), 275706.

Hirata, M., Gotou, T., Horiuchi, S., Fujiwara, M., \& Ohba, M. (2004). Thin-film particles of graphite oxide 1:: High-yield synthesis and flexibility of the particles. Carbon, 42(14), 2929-2937.

Kohn, W., \& Sham, L. J. (1965). Self-consistent equations including exchange and correlation effects. Physical review, 140(4A), A1133.

Lima, K. A. L., Júnior, M. L. P., Monteiro, F. F., Roncaratti, L. F., \& Júnior, L. A. R. (2021). O2 adsorption on defective Penta-Graphene lattices: A DFT study. Chemical Physics Letters, 763, 138229.

Neto, A. C., Guinea, F., Peres, N. M., Novoselov, K. S., \& Geim, A. K. (2009). The electronic properties of graphene. Reviews of modern physics, 81(1), 109.

Nguyễn Thành Tiên \& Trần Yến Mi. (2020). Nghiên cứu hiện tượng hấp phụ phân tử khí trên dãy nano Penta-graphene dạng răng cưa. Tạp chi khoa hoc Truòng Đại học Cần Tho, 56(2), 21-29.

Perdew, J. P., Burke, K., \& Ernzerhof, M. (1996). Generalized gradient approximation made simple. Physical review letters, 77(18), 3865.

Tien, N. T., Thao, P. T. B., Phuc, V. T., \& Ahuja, R. (2019). Electronic and transport features of sawtooth penta-graphene nanoribbons via substitutional doping. Physica E: Low-

dimensional Systems and Nanostructures, 114, 113572.

Wang, Z., Dong, F., Shen, B., Zhang, R. J., Zheng, Y. X., Chen, L. Y., \& Su, W. S. (2016).

Electronic and optical properties of novel carbon allotropes. Carbon, 101, 77-85.

Yu, Z. G., \& Zhang, Y. W. (2015). A comparative density functional study on electrical properties of layered penta-graphene. Journal of Applied Physics, 118(16), 165706.

Zhang, S., Zhou, J., Wang, Q., Chen, X., Kawazoe, Y., \& Jena, P. (2015). Penta-graphene: A new carbon allotrope. Proceedings of the National Academy of Sciences, 112(8), 2372-2377. 